\title{
Endosonografie (EUS)-gestützte transluminale Cholangiodränage (EUCD) - eine neue Option der interventionellen Endoskopie im interdisziplinären Management des obstruktiven Ikterus
}

\author{
Endoscopic Ultrasonography (EUS)-Guided Transluminal Cholangiodrainage (EUCD) - \\ A Novel Option of Interventional Endoscopy in the Interdiciplinary Management of Obstructive Jaundice
}

Autoren

Institute

\section{U. Will' ${ }^{1}$ F. Meyer ${ }^{2}$}

${ }^{1}$ SRH Wald-Klinikum Gera gGmbH, Klinik für Gastroenterologie, Hepatologie \& Allgemeine Innere Medizin, Gera, Deutschland

${ }^{2}$ Universitätsklinikum Magdeburg A. ö. R., Klinik für Allgemein-, Viszeral- \& Gefäßchirurgie, Magdeburg, Deutschland

\author{
Schlüsselwörter \\ - interventionelle Endo- \\ sonografie (EUS) \\ - Gastrointestinal(Gl)-Trakt \\ - Gallengang \\ - Papilla Vateri \\ - EUS-geführte Cholangio- \\ dränage (EUCD) \\ - ERCP/PTCD

\section{Key words} \\ - interventional endoscopic \\ ultrasonography (EUS) \\ - gastrointestinal(Gl) tract \\ - bile duct \\ - papilla of Vater \\ - EUS-guided cholangio- \\ drainage (EUCD) \\ - ERCP/PTCD
}

\section{Zusammenfassung}

$\boldsymbol{\nabla}$

Hintergrund: ERCP und PTCD gelten als der Goldstandard für die interventionelle Behandlung der biliären Obstruktion, insbesondere unter palliativer Intention. Unter bestimmten Bedingungen sind eine ERCP als auch PTCD nicht möglich. In diesen Fällen können alternative Entlastungsverfahren wie beispielsweise eine endosonografisch gestützte Cholangiodränage zum Einsatz kommen.

Ziel / Methode: Anhand einer kompakten Kurzübersicht sollen Indikation, Technik, Varianten des Herangehens, Behandlungszahlen verschiedener Autoren, Erfolgsrate, Komplikationsspektrum und -management als auch Nachsorgeempfehlungen zur EUS-geführten Cholangiodränage (EUCD) auf der Basis eigener klinischer Erfahrungen als auch im Vergleich zu publizierten Daten umrissen werden.

Ergebnisse: Die EUCD ist ein interventionellendoskopisches/endosonografisches Verfahren, das bei veränderter Oberbauchanatomie nach Vor-OP (BII-Magenresektion, PPPHR, WhippleOP, [sub-]totale Gastrektomie, Roux-en-Y-Rekonstruktion) und damit Nicht-Erreichbarkeit bzw. -Sondierbarkeit der Papilla Vateri (Papille) sowie bei PTCD-Ablehnung beim rezidivierenden, fortgeschrittenen oder metastasierten malignen Tumorgeschehen mit assoziierter Gallenwegsobstruktion und Ikterus Anwendung findet. Vom Prinzip her wird mittels EUS-geführter transluminaler Punktion vom oberen GI-Trakt aus in verschiedene extra-/intrahepatische Gallenwegssegmente die Tumorstenose mittels Stent rekanalisiert oder unter Tumorumgehung der stentbasierte Galleabfluss retrograd oder antegrad herbeigeführt. Daraus leiten sich Zugänge und Vorgehen ab: 1.Rendezvous-Technik, 2.transhepatisch mit retrograder (permanente HepaticoEnterostomie) oder 3.transtumoröse antegrade interne Dränage, 4. extrahepatisch mit antegrader

\section{Abstract \\ $\nabla$}

Background: ERCP and PTCD are considered the gold standard in the interventional treatment of biliary obstruction, in particular, with palliative intention. If ERCP and PTCD are not possible, an alternative drainage procedure such as the EUSguided cholangiodrainage (EUCD) can be used.

Aim / Method: By the mean of a compact review, indication, technique, variants of approach, number of treated patients and therapeutic procedures reported by various authors, success rate, spectrum and management of complications as well as recommendations for an appropriate follow-up-investigation protocol for EUCD based on our own clinical experiences and compared to published data are described.

Results: EUCD is an interventionally endoscopic/ -sonografic procedure, which is used in case of postoperatively changed anatomy of the upper GI tract (BII gastric resection, PPPHR, Whipple procedure, [sub-]total gastrectomy, Roux-en-Y reconstruction) and, thus, if papilla of Vater (papilla) can not be reached or catheterized or if the patient denies PTCD in subjects with recurrent, advanced or metastasized tumor lesion(s) of the upper abdomen, hepatobiliary system as well as pancreas and associated obstruction of the biliary tree $-1+$ jaundice. Principle: EUS-guided transluminal puncture from the upper GI tract into various extra-/intrahepatic segments of the biliary system, recanalization of the tumor stenosis with stent insertion through the access site or bypassing the tumor (stent-based retro- or antegrade drainage of the biliary tree). Derived from this, there are various approaches and procedures - EUCD i) combined with rendesvouz technique, ii/iii) transhepatically with retro- (permanent hepaticoenterostomy)/antegrade internal drainage, iv) extrahepatically with antegrade drainage (permanent choledocho-enterostomy), which are distinguished according to tumor site, 
Galleableitung (permanente Choledocho-Enterostomie), die je nach Obstruktion zu favorisieren sind. Im Komplikationsspektrum (Rate Lit.: $_{0}$-25\%) sind Blutung, Perforation, Stentdislokation/-migration/-okklusion sowie moderater postinterventioneller Schmerz zu nennen. Derzeit sind mehr als 200 Fälle in der Welt publiziert, wobei sich der eigene Erfahrungswert auf $>70$ Interventionen stützt.

Diskussion: Im derzeitigen Verbreitungsgrad der EUCD ist noch nicht von einem Standardverfahren auszugehen. Als Vorteile sind das meist geringe Gewebstrauma, die primäre interne Dränage und die endoskopisch mögliche Reintervention bei Komplikationen zu nennen. Nachteilig fällt der hohe technische Anspruch für eine breitere Anwendung ins Gewicht. Bei ausweisbarer Erfolgsaussicht im Rahmen offen gelegter EUCD-Behandlungsergebnisse kann die EUCD bereits durchaus als kompetitives Verfahren zu ERCP und PTCD betrachtet werden.

Schlussfolgerung: Die EUCD ist eine sich etablierende, allerdings noch immer experimentelle, interventionell-endoskopische/-endosonografische Prozedur, die eine hohe Expertise des Interventionalisten im interdisziplinären Verbund eines viszeralmedizinischen Zentrums voraussetzt. Sie stellt eine elegante, in erfahrener Hand sichere Vorgehensweise dar, die jedoch noch immer ein systematisches Follow-up sowie eine multizentrische Evaluierung bis hin zur möglichen Leitlinienrelevanz erfordert. possible direction of translumenal puncture, insertion of a guide wire and final stent placement. Within the spectrum of complications (rate Lit. $_{0}$ 0-25\%), bleeding, perforation, stent dislocation/ -migration/-occlusion and slight postinterventional pain are relevant. Currently, approximately 200 cases have been published worldwide; the clinical experience of the reporting institution is based on more than $>70$ interventions.

Discussion: With regard to the limited diffusion process, EUCD cannot be considered a standard procedure yet. The advantages comprise low tissue trauma, primary internal drainage and the possible endoscopic re-intervention in case of complications. The high technical challenge in performing EUCD is a disfavourable aspect for broader use in clinical practice. However, the disclosed treatment results demonstrating an acceptable complication rate show that EUCD can be competitively considered to ERCP und PTCD with a great chance for primary success.

Conclusion: EUCD is an elegant, not yet fully established, but rather still experimental procedure of interventional endoscopy / EUS, which needs great expertise of the endoscopist in an interdisciplinary centre of visceral medicine as one of the main predictions. In experienced hands, a safe procedure can be provided, for which a systematic follow-up and a multicentre evaluation of periinterventional management are still needed in order to achieve a final assessment of EUCD for guideline approval.

\section{Einleitung}

Die transluminale Cholangiodränage (Synonym: EUS-gestützte [transluminale] Cholangiodränage [EUCD]) stellt eine neue, alternative Herangehensweise der suffizienten, interventionell- endoskopischen und damit minimalinvasiven Versorgung der Obstruktion von Gallenwegen dar. Die Methode kann bei erfolglosem Versuch der endoskopischen Dränage via ERC oder bei Problemen bzw. Kontraindikationen zur PTCD zum Einsatz kommen [1 -7]. Grundlage für den erfolgreichen Einsatz dieser neuen Dränagetechnik ist neben einem hohen Level der klinischen Erfahrung, die eine adäquate Indikationsstellung einschließt, v.a. eine außerordentliche Expertise im interventionell-endoskopischen und -endosonografischen Handling.

Neben der differenzialtherapeutischen, endoskopisch / -sonografisch basierten Therapie von Pankreaspseudozysten, -abszessen und -nekrosen $[8,9]$ sowie der EUS-gestützten, teils mit ERP kombinierten Pankreasgangdränage (EUPD) [2, 3, 10-13] beginnt sich damit ein weiterer Meilenstein der durchaus als NOTES zu betrachtenden interventionellen Endoskopie und -sonografie im klinischen Alltag zu etablieren.

Das Ziel der vorliegenden kompakten Kurzübersicht ist es, die technischen Möglichkeiten in Korrelation zu den spezifischen klinischen Konstellationen darzustellen und unter den Gesichtspunkten

- technische Machbarkeit,

- klinische Erfolgsrate,

- Komplikationsrate sowie

- Limitationen und Grenzen

der EUS-gestützten Cholangiodränage ihre Relevanz für den klinischen Alltag bei entsprechender Expertise eines Zentrums zu charakterisieren sowie unter Einbeziehung aktueller Literaturdaten kritisch zu würdigen [14].

\section{Pathoanatomischer /-physiologischer Hintergrund - Indikationen}

$\nabla$

Die endoskopische retrograde Cholangiodränage (ERC) oder alternativ bei Unmöglichkeit des transpapillären Zuganges die perkutane transhepatische Cholangiodränage (PTCD) gelten als etablierte Methoden der Wahl und als Goldstandard bei maligner biliärer Obstruktion [15-20] zur Anlage einer Cholangiodränage.

Es gibt nicht selten klinische Konstellationen und fallabhängige Umstände, in denen beide Methoden (nach sequenziellem Vorgehen) versagen, nicht angezeigt sind oder aber auch abgelehnt werden. Insbesondere die PTCD mit der psychologischen Schwelle einer zu akzeptierenden extrakorporalen Dauerdränage der Gallenwege mit dem immanenten Komplikationspotenzial (Lokalinfektion, Leckage, Galle-/Flüssigkeits-/Elektrolytverlust etc.) sind für ein palliatives Behandlungskonzept mit dem Anspruch „Primum nihil nocere“ eher ungeeignet.

Im Falle

- einer nicht erreichbaren Papilla Vateri (Papille) aufgrund:

- einer Pylorus- oder Duodenalstenose (Magenausgangsstenose),

- eines Tumorrezidivs bzw. fortgeschrittenen Tumorwachstums im Magen oder Duodenum, oder

- eines früheren operativen Eingriffs mit Veränderung der Oberbauchanatomie (B-II-Magenresektion, OP nach Kausch-Whipple, (sub-)totale Gastrektomie mit Roux-en-YRekonstruktion),

- eines nicht einführbaren Katheters in die Papille respektive in den gestauten Gallengang wegen:

• postinflammatorischen Veränderungen der periampullären Region

oder 
$>$ maligner Tumorläsion

- der Papille,

- des Pankreas oder

- des Gallenwegsystems,

wird die PTCD als alternative Therapieoption bei Cholestase anstelle der nicht erfolg- bzw. aussichtsreichen therapeutischen ERCP angesehen [18 - 25].

Die PTCD ist im Vergleich mit der ERC mit einer höheren Komplikationsrate belastet und zudem mit den Problemen

- der existenten, wenn auch niedrigen Rate an Fehlpunktionen mit Hämobilie, Bilhämie,

- einer nicht immer erreichbaren extern-internen Dränage bei Unmöglichkeit der Überwindung der Tumorstenose mit der Folge einer alleinigen externen Dränage mit Galleverlustsyndrom und

- eines für manche Patienten immens bedeutsamen psychologischen Aspekts einer nach extrakorporal erfolgenden Katheterableitung des Galleabflusses

behaftet [18-20].

\section{Vorbetrachtungen \\ $\nabla$}

Die endoskopische Sonografie (EUS) hat sich durch ihre Kombination der Endoskopie mit der hochauflösenden transmuralen Sonografie von ihrem bis in die Mitte der 90er-Jahre dominierenden alleinigen diagnostischen Stellenwert gelöst und sich hin zu einer therapeutischen Methode entwickelt, deren Potenzial derzeit weiter erforscht und vervollkommnet wird [1-7, 14, 26].

Das Profil EUS-gestützter Therapieansätze ist dabei während der letzten Dekade einem enormen Wandel hinsichtlich der Erweiterung bzw. Modifizierung des endoskopisch-interventionellen Spektrums unterworfen worden [14].

Diese interventionell-endoskopischen Therapieerweiterungen können die alternativen operativen Interventionen mit der traumatischen Invasivität mit allen Komplikationen deutlich limitieren und damit eine Risiko-Nutzen-Relation gerade für das risikoträchtige Patientenklientel unter palliativer Intention eines malignen Grundleidens weit günstiger und v.a. sicherer gestalten [14, 27, 28].

\section{Rationale und Voraussetzungen}

Die zunehmende Zahl von palliativ zu behandelnden Patienten induziert das Erfordernis, auf die manigfaltigen klinischen Konstellationen, die mit einer Gallenwegsobstruktion ob von endoluminal oder durch Obstruktion von außen einhergehen, flexibel und falladaptiert zu reagieren.

Es muss der medizinische Anspruch sein, eine optimale und risikoarme Versorgung gerade in der Situation einer limitierten Lebenserwartung für die Patienten mit malignem Ikterus herbeizuführen, die den Vorgaben

- der Ausführbarkeit,

- der Gewährleistung der Vorteile einer (schonenden) minimalen Invasivität,

- der Erreichung einer internen und permanenten Galleableitung,

- des limitierten Komplikationspotenzials,

- der endoskopischen Reinterventionsoption,

- der ökonomischen Tragfähigkeit und

- der Wahrung einer gebesserten Lebensqualität (trotz Palliation)

gerecht wird $[29,30]$.
Als allgemeiner Vorteil der EUCD im Vergleich zur PTCD ist die Vermeidung einer dauerhaften bzw. transienten (externen) Gallenwegsdränage aufzuführen. Neben den Komplikationen einer Leckage mit konsekutiven Hautinfektionen kann v.a. das psychologische Moment der Verletzung der körperlichen Integrität mit der sichtbaren Dränage als stetig erinnernder Hinweis auf die Inkurabilität vermieden werden. Daneben wird es aufgrund des „internen“ Galleableitungsverfahrens durch die EUCD möglich, eine endoskopische Reintervention im Falle von Stentobstruktionen oder anderen Problemen mit der Dränage ausführen zu können. Der Eingriff ist in diesem Fall vergleichbar mit einer ERCP ohne die gefürchtete Komplikation einer Pankreatitis [29].

Als Nachteile sind

- der hohe technische Anspruch in der Ausführung (Kombination von Endoskopie und interventioneller EUS sowie ERC, versiertes endoskopisches Komplikationsmanagement, hohe technische Skills des Endoskopieteams, technische (teils kostenintensive) Ausstattung,

- der notwendige Hintergrund eines interdisziplinären Zentrums (Viszeralchirurgie, interventionelle Radiologie)

anzuführen [23].

Das hohe Problempotenzial im Falle einer unausweichlich notwendig werdenden Operation bei endoskopisch nicht beherrschbaren Komplikationen (z.B. Stentmigration in die Bauchhöhle) erfordert den ebenso erfahrenen und versierten viszeralchirurgischen Partner des federführenden interventionell-endoskopisch tätigen Gastroenterologen, um die dann verloren gehenden Vorteile des schonenden endoskopischen Vorgehens (s.o.) durch die erforderlichen OP-Konsequenzen (Invasivität, Narkose- / OP-Trauma, OP-eigenes Komplikationsprofil) durch ein gezieltes Vorgehen mit begrenzter Invasivität ausgleichen zu helfen [21-25].

\section{Technische Ausführung - eigenes Vorgehen [14]}

Nach einer eingehenden perkutanen Sonografie durch den Endosonografiker, bei der man sich über die Lokalisation der Obstruktion und das Ausmaß der Cholestase als auch über mögliche EUSZugangswege ein Bild verschafft, erfolgt die adäquate Aufklärung des Patienten über den Eingriff inkl. Komplikationen, Alternativen und ggf. einer notfallmäßigen PTCD oder OP-Konsequenz. Eine periinterventionelle Applikation von gallenwegsgängigen Antibiotika (z. B. Cephalosporine der 3. Generation) wird in allen Fällen durchgeführt. Dies dient v.a. der Abschirmung des Patienten vor einer Keiminvasion während der Intervention an den gestauten Gallengängen, die in mehr als 50\% im eigenen Patientenklientel in der obligatorischen mikrobiologischen Untersuchung ein pathologisches Keimspektrum aufwiesen.

Der Patient wird einer EUS mit einem therapeutischen Longitudinalscanner (Hitachi Medical Systems GmbH, Wiesbaden, Olympus Europa GmbH, Hamburg) auf einem Fluoroskopietisch unterzogen, wenn eine konventionelle ERCP nicht gelungen war oder diese nicht möglich ist. Nach Identifikation der gestauten Gallenwege und in Kenntnis der Obstruktionshöhe und der Anatomie wird ein zentraler intrahepatischer Gallengang (geplante Hepatikoenterostomie) oder der Ductus hepatocholedochus (geplante Choledocho-Enterostomie) mit einer 19-G-Nadel (Wilson-Cook Deutschland, Mönchengladbach, Deutschland) punktiert, Gallenflüssigkeit wird aspiriert (simultan initiierte mikrobiologische Kultur) und der Gang mittels kontrastmittelverstärkter Fluoroskopie dargestellt. Ein 0,035-Inch-Führungsdraht (Boston Scientific, 
Ratingen, Deutschland) wird soweit wie möglich in die Gallenwege eingeführt. Über den Draht wird ein HF-Ringmesser (MTWEndoskopie W.Haag KG, Wesel, Deutschland) transluminal eingebracht und in Einzelfällen (derbes Leberparenchym, weiter Weg) eine Ballondilatation ausgeführt. In Abhängigkeit von der fallspezifischen Anatomie und der Art des Herangehens wird wahlweise ein „gecoverter“ Metallstent (Boston Scientific, Ratingen, Deutschland) oder eine 8,5-French-Pigtail-Plastikprothese (Endoflex, Voerde, Deutschland) oder eine 8,5- oder 10-Frenchgerade Amsterdamprothese (Medi-Globe, Achenmühle, Deutschland) unter EUS- und Endoskopiesicht platziert. Eine obligatorische Kontrolle der Lage und der Funktion des Stents erfolgt mit transabdomineller Sonografie am ersten postinterventionellen Tag. Hier achtet man auf eine Aerobilie als Hinweis auf eine regelrechte Funktion und auf die Länge des intraluminalen Stentanteils im Falle, dass ein selbstexpandierender Metallstent platziert wurde. Letzteres ist wichtig, da sich hieraus bereits präferentiell ableiten lässt, ob mit einer Stentmigration zu rechnen ist.

In mehreren Arbeiten zur EUCD kristallisieren sich 4 Techniken der Dränage heraus, die in Abhängigkeit von der Anatomie (Papille erreichbar, Z.n. Operation) und dem Verschlusstyp (proximaler/distaler Verschluss) zum Einsatz kommen und im Weiteren nach der Reihenfolge des empfohlenen Vorgehens aufgeführt sind:

\section{EUCD in Rendezvoustechnik}

Patienten mit Verschlussikterus, bei denen die Papille erreichbar, der Gallengang aber infolge Tumorobturation oder Papillendeformation nicht zu intubieren ist, sind ideale Kandidaten für eine EUS-gestützte Dränage in Rendezvoustechnik. Nach gezielter transduodenaler, tangentialer Punktion des DHC oder bei hoher Obstruktion einer Punktion des linken intrahepatischen Gallenwegssytems (Gallengang 3. Ordnung) mit einer 19-G-Nadel wird ein 0,035-Inch-Draht eingebracht und dieser aus der Papille ausgeleitet $(\bullet$ Abb.1, 2).

Nach Punktion des linken D. hepaticus muss vorher noch der Tumor passiert werden. Dies gelingt nicht immer und sollte nicht forciert werden, da alternativ eine EUS-geführte retrograde Dränage möglich ist. Gelingt die Überwindung der Tumorstenose und die Drahtausleitung aus der Papille, erfolgt der Gerätewechsel auf ein therapeutisches Duodenoskop. Der Draht wird aufgenommen und ausgeleitet und die ERC in klassischer Weise durchgeführt. Die Vorteile einer EUS-gestützten Rendezvoustechnik im Vergleich zur alternativen PTCD nach frustraner ERC liegen darin, dass unmittelbar nach der erfolglosen ERC mithilfe der EUS eine gezielte Punktion der Gallenwege erfolgen kann und die ERC nach Drahtaufnahme in konventioneller Technik zu beenden ist. Eine Zweituntersuchung kann dem Patienten erspart werden, die interne orthograde Dränage gelingt in einer Sitzung. Die Komplikationsrate ist niedrig, da nach Punktion der Gallenwege außer einer Drahteinführung keine weiteren Manipulationen stattfinden [31].

\section{EUCD transhepatisch mit antegrader interner Dränage} Patienten mit malignem Verschlussikterus, bei denen die Papille nicht erreichbar ist durch:

a) Z.n. Operationen (B-II, Roux-en-Y-Rekonstruktion des oberen GI-Trakts, [sub-]totale Gastrektomie, pankreato-duodenale Diversion, Hepatikojejunostomie) oder

b) maligne Magenausgangsstenosen (Magen-, Pankreas-, Cholangio- und Gallenblasenkarzinom),

können mit zwei verschiedenen Techniken behandelt werden. Bei den Patienten handelt es sich meist um Obstruktionen der

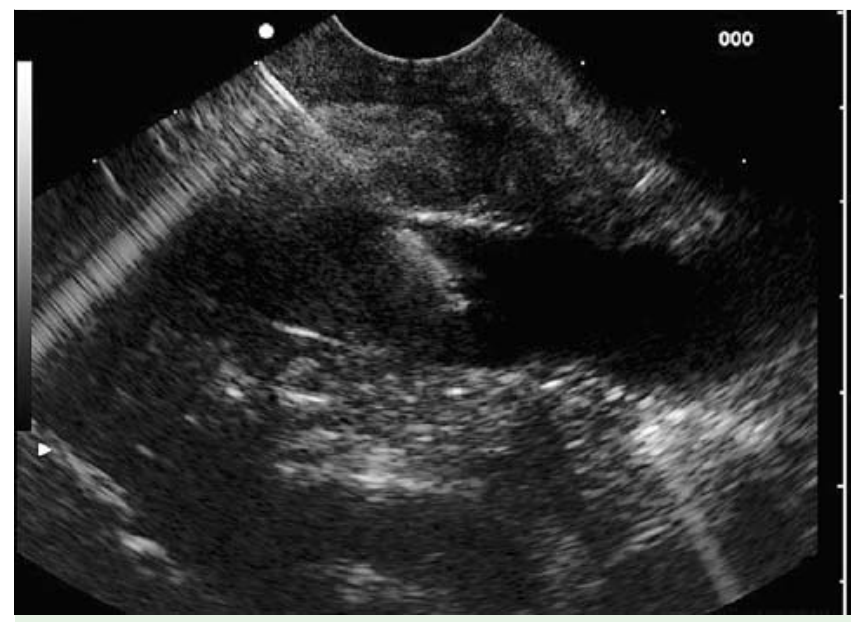

Abb. 1 DHC in ERC nicht sondierbar, daher transduodenale Punktion des D. choledochus mit 19-G-Nadel.

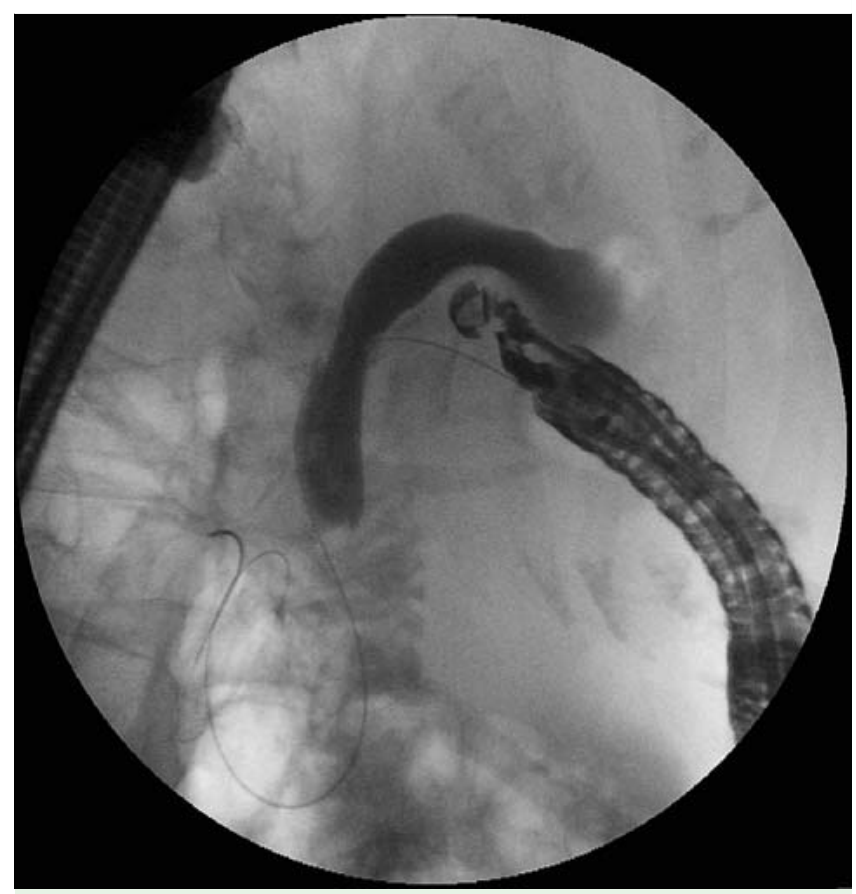

Abb.2 Ausleitung des Drahtes aus der Papille ins Duodenum und Gerätewechsel auf das Duodenoskop.

Gallenwege durch Tumorrezidive von gastrointestinalen oder biliopankreatischen Tumoren, bei denen eine Reoperation in kurativer Absicht nicht angezeigt ist, oder um Patienten mit primär inkurablen Befunden (Metastasierung, Komorbidität, lokal fortgeschrittenes Tumorwachstum). Die Punktion des Gallenwegsystems im linken Leberlappen erfolgt transhepatisch je nach anatomischer Situation über den Ösophagus, den Magen oder das Jejunum (z.B. hochgezogene Jejunalschlinge) ( $\triangle$ Abb.3). Gelingt die Überwindung der Tumorstenose mit einem 0,035-Inch-Draht, kann ggf. nach HF-Diathermie oder Ballondehnung der Tumorstenose ein Metallstent tansluminal-transhepatisch eingebracht werden, der die Tumorstenose intern im Gallenwegsystem überbrückt ( $\bullet$ Abb.4). Gelingt keine Drahtpassage des Tumors, kann eine retrograde Ableitung des Gallenwegsystems erfolgen (transhepatische, retrograde EUCD).

Die Vorteile der transhepatischen antegraden internen Dränage im Vergleich zur PTCD bei nicht erreichbarer Papille liegen darin, 


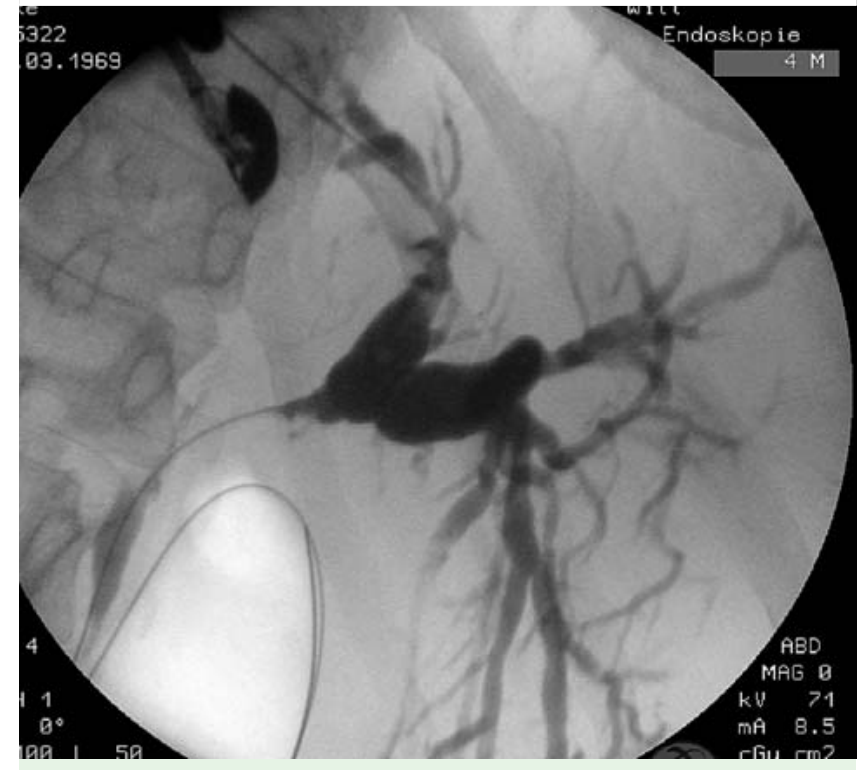

Abb.3 Patientin mit obstruktivem Ikterus bei metastasiertem Mammakarzinom, EUS-Punktion des linken D. hepaticus, Drahtausleitung aus der Papille.

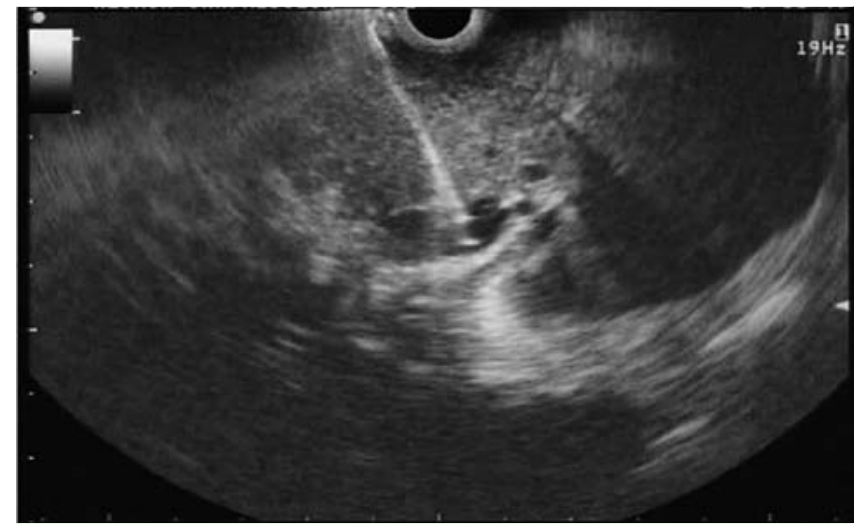

Abb.5 Obstruktion bei Lokalrezidiv nach Gastrektomie bei Magenkarzinom; transjejunale EUS, Punktion des linken D. hepaticus.

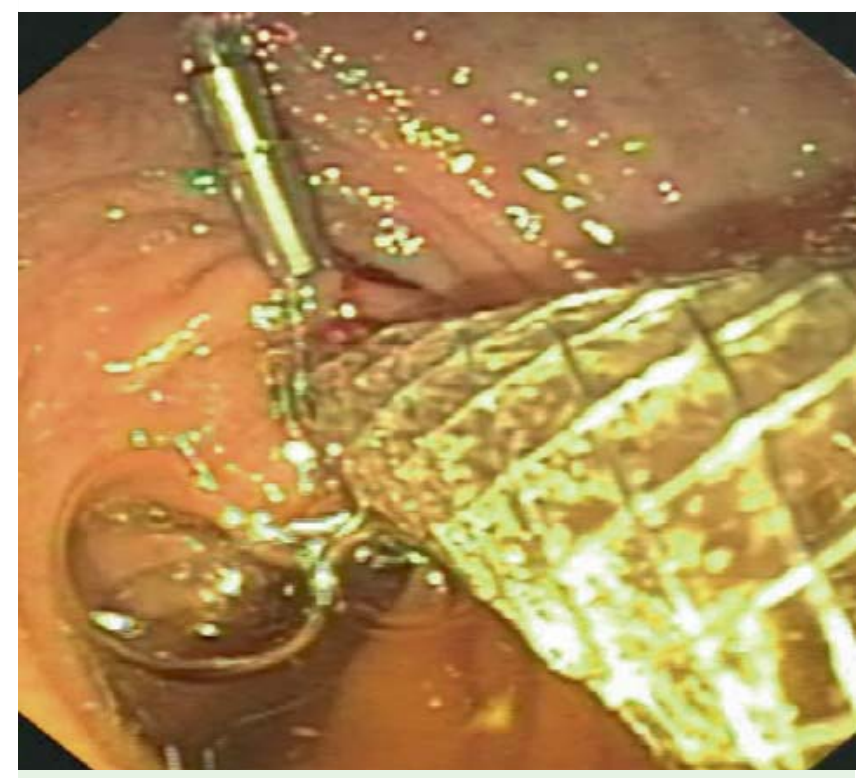

Abb.7 Fixation des SEM durch Metallclip.

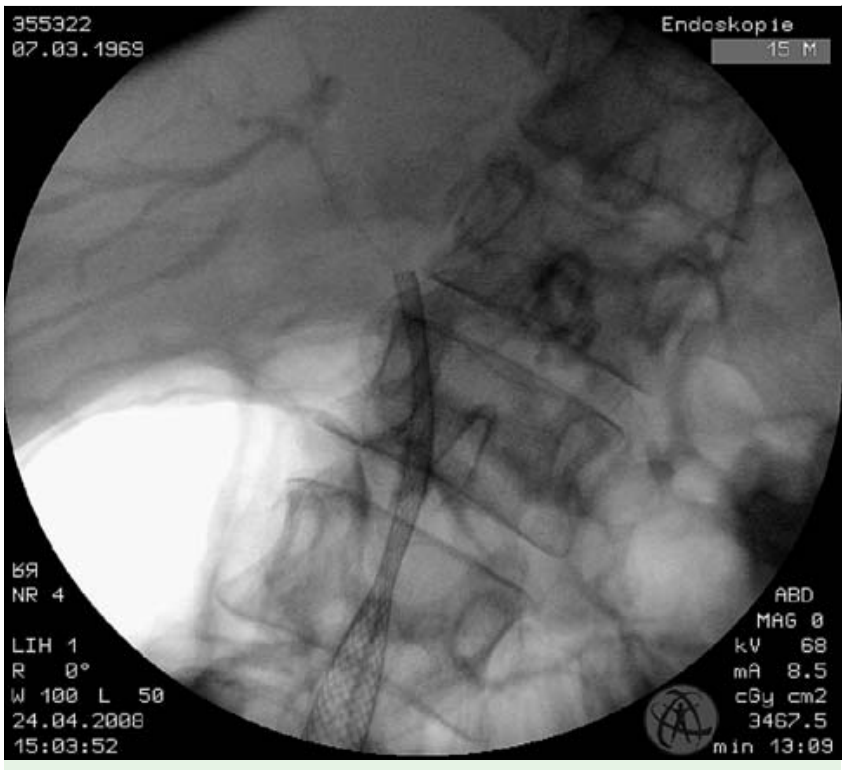

Abb.4 Nach transhepatischer Einbringung eines gecoverten Metallstents - Aerobilie.

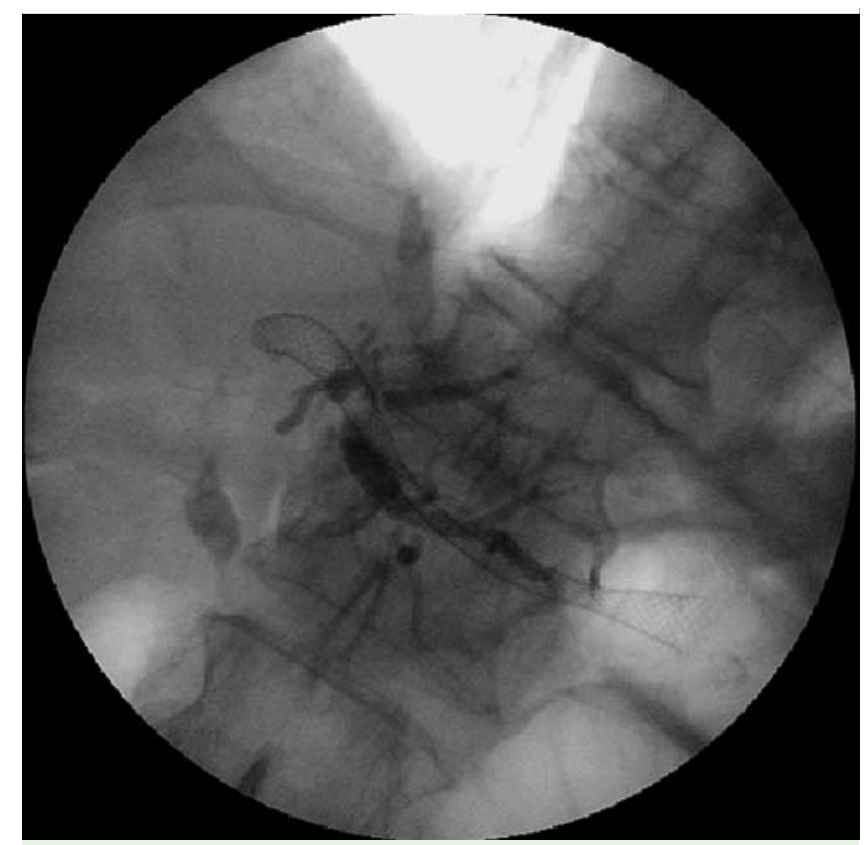

Abb.6 Freisetzung eines SEM.

dass eine interne Galleableitung ohne perkutane Intervention möglich ist. Die Punktion der Leber erfolgt von intestinal, sodass dieses Vorgehen komplikationsärmer zu sein scheint. Die gefürchtete Stentdislokation wie bei retrograden Galleableitungen ist mit dieser Technik auszuschließen [32].

Nachteil dieser Technik ist, dass die Intervention an der Tumorstenose in jedem Fall eine Drahtpassage voraussetzt. Dies ist technisch nicht immer möglich - in diesen Fällen muss auf eine retrograde Hepatico-Intestinostomie ausgewichen werden.

\section{EUCD transhepatisch mit retrograder Galleableitung} (permanente Hepatico-Enterostomie)

Indikationen für dieses endosonografische Dränageverfahren sind Patienten mit maligner Obstruktion, bei denen die Papille 

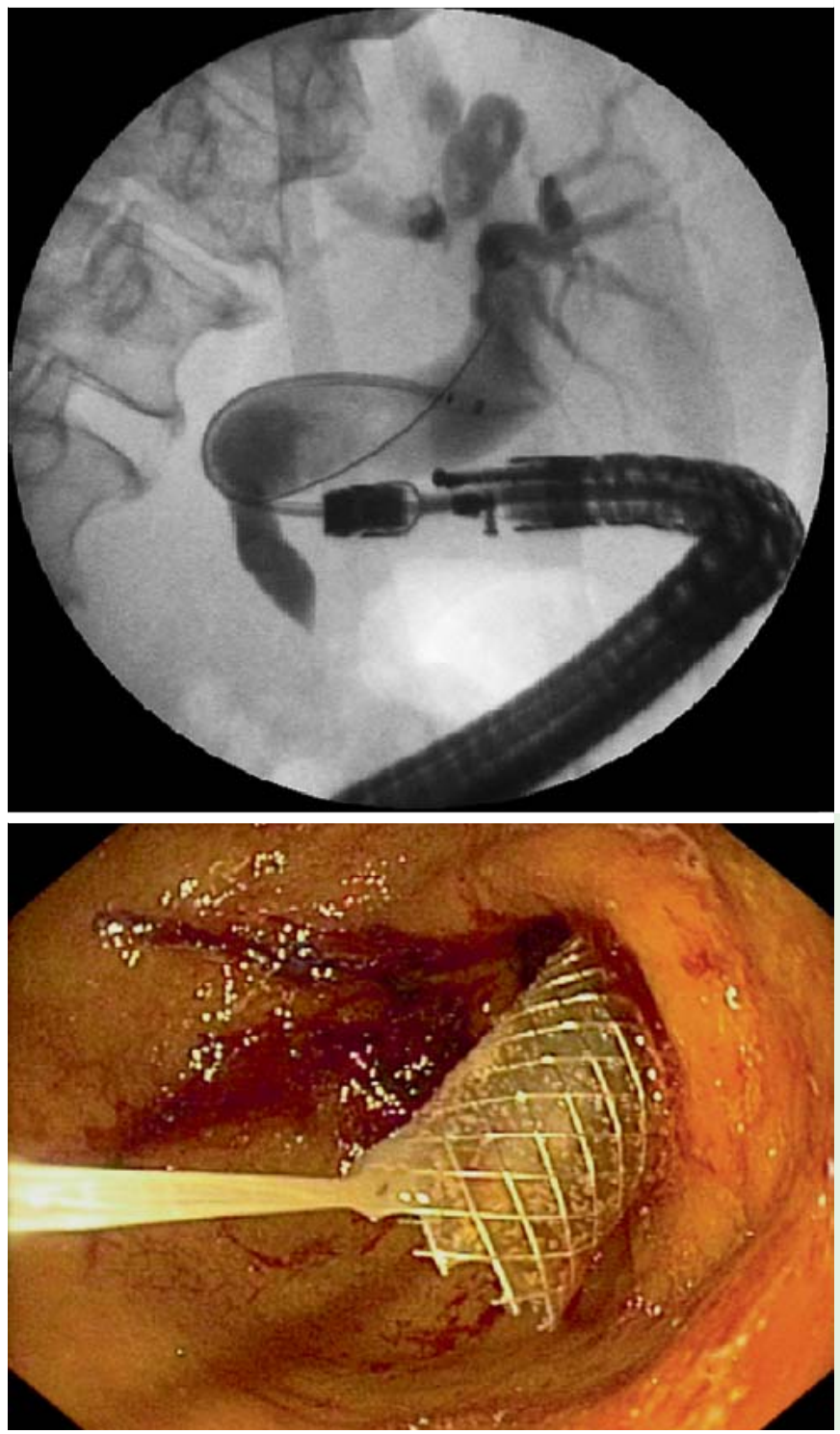

nicht mehr erreichbar ist und der Tumor im Gallenwegsystem nicht zu überwinden oder dieser per continuitatem in das intestinale Lumen eingebrochen ist. In diesen Fällen sollte eine antegrade interne Dränage vermieden werden, da eine Tumorobturation durch Einwachsen droht, wenn das untere Stentende im Tumor positioniert wird. Die Intervention beginnt mit einer transhepatischen Punktion eines im Querschnitt darzustellenden zentralen Gallenganges im linken Leberlappen. Nach Cholangiografie und Drahtvorschub erfolgt je nach Anatomie der Gangsysteme die Einbringung eines gecoverten Metallstents oder einer Pigtaildränage ( $\bullet$ Abb.5, 6). In Einzelfällen ist nach regelhafter Konditionierung des Zuganges mit HF-Ringmesser eine Ballondilatation vor Stentplatzierung erforderlich. Die Einlage des gecoverten Metallstents hat den Vorteil eines großen Lumens und - nach Freisetzung - einer sofortigen Abdichtung des Enterostomieostiums. Nachteile sind die Okklusion intrahepatischer Nebengänge und die hohe Dislokationsgefahr. Letzteres kann man durch endoskopische Applikation von luminalen Clips vermeiden ( $\bullet$ Abb.7). Eine endoskopische Reintervention ist im weiteren Verlauf bei Dränageproblemen möglich [4, 33].

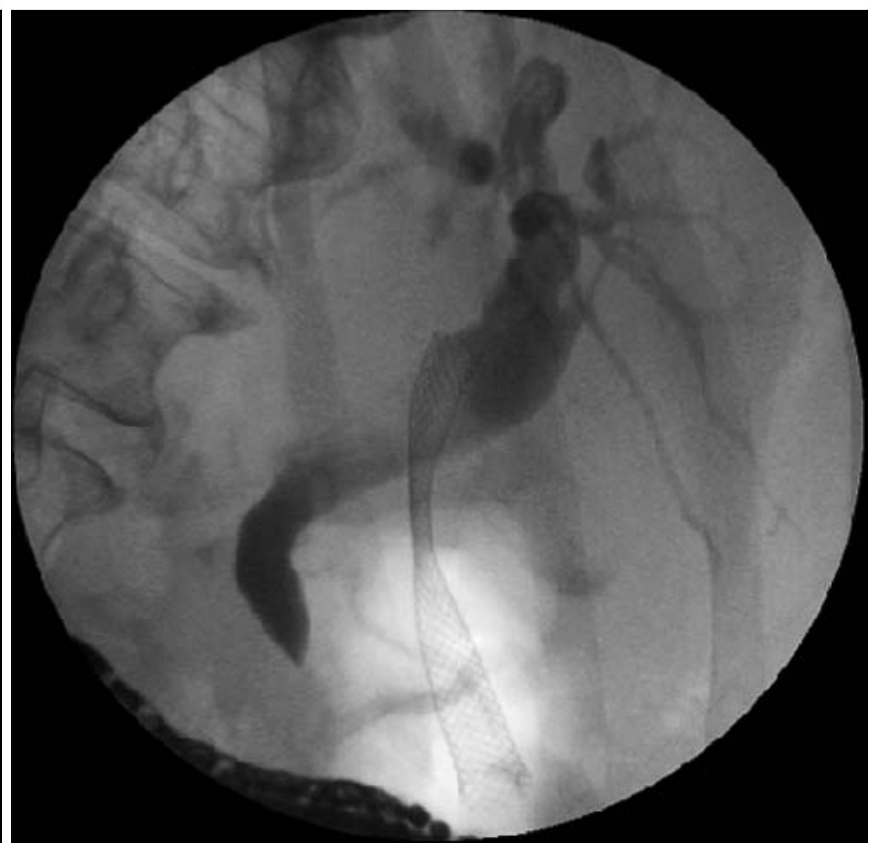

Abb.8-10 Patient mit fortgeschrittenem Duodenalkarzinom mit Peritonealkarzinose, Z.n. Gastroenterostomie - Papille im Tumor; transgastrische Punktion des D. choledochus mit 19-G-Nadel; Zugang über Draht mit HFRingmesser, Einlage eines gecoverten SEM.

\section{EUCD extrahepatisch mit antegrader Galleableitung} (permanente Choledocho-Enterostomie)

Bei Patienten mit malignem peripapillären Verschlussikterus (Metastasierung, Papillen-, distales Pankreas- oder Cholangiokarzinom bzw. maligner Magenausgangs- oder Duodenalstenose), bei denen eine extra- und intrahepatische Cholestase vorliegt, die Papille allerdings nicht mehr erreichbar ist, kann eine extrahepatische antegrade EUS-gestützte Dränage neben der besprochenen retrograden transhepatischen Dränage versucht werden. Hierbei wird endosonografisch der Choledochus tangential eingestellt, wobei die Punktion entgegen der Rendezvoustechnik in Richtung Leberhilus ausgeführt werden sollte ( $\bullet$ Abb.8). Die Punktion erfolgt je nach anatomischer Situation aus dem Bulbus oder dem Antrum. Nach Punktion mit der 19-G-Nadel und einer Cholangiografie wird ein 0,035-Inch-Draht eingeführt. Die Choledocho-Enterostomie wird über ein HF-Ringmesser, in Einzelfällen gefolgt von einer Ballondehnung, für die Stentinsertion vorbereitet. Präferenziell sollten bei der extrahepatischen antegraden Dränage gecoverte SEM zum Einsatz kommen, da neben einem sicheren Verschluss der Enterostomie eine stabile Lage der Dränage bei weitem Lumen erreichbar ist ( $\bullet$ Abb.9, 10). 
Um eine Dislokation zu vermeiden, ist die luminale Fixation des Stents mit Metallclips sinnvoll. Vorteil der antegraden extrahepatischen Choledocho-Enterostomie ist die Schaffung eines Neoostiums mit der Möglichkeit einer Reintervention am Gallengang, eine fehlende Obturation von Seitenästen und einer geringeren Dislokationsgefahr [5].

\section{Komplikationen \\ $\nabla$}

Die Komplikationsraten der EUS-gestützten Cholangiodränage werden in der Literatur mit 0-25\% angegeben [4, 7, 30, 33 -44]. Eingriffsimmanent sind luminale Blutungen und Einblutungen in das Gallenwegsystem sowie seltener Perforationen oder akute Stentdislokationen. Daneben sind Stentmigrationen/-occlusionen als auch Cholangitiden im kurz- bis mittelfristigen postinterventionellen Verlauf zu nennen. Ein leichter postinterventioneller Schmerz sowie ein temporäres Pneumoperitoneum scheinen nach eigenem Erfahrungswert bei ca. 20 - 30\% der Patienten vorzukommen. Im unmittelbaren postoperativen Verlauf verlieren sich sowohl der Schmerz als auch die Spuren freier Luft. Der Schmerz ist durch Analgetika gut zu kopieren [1, 4-6, 10, 34, 35]. Die akute Stentmigration eines SEM in die freie Bauchhöhle nach Freisetzung ist ein seltenes, aber dramatisches Ereignis. Die folgenden Interventionen sollten neben der Stentbergung und dem sicheren Verschluss des Intestinums auch eine sichere externe Ableitung der Gallenwege mit PTCD beinhalten. Eine späte Stentdislokation und -okklusion sind weitere Probleme, die immer in erster Linie endoskopisch angegangen werden.

Interventionsassoziierte Todesfälle sind bei bisher mehr als 200 Anwendungen die Ausnahme geblieben. Dies ist v.a. der verantwortungsvollen Indikationsstellung und der kompetenten Expertise der Anwender zuzuschreiben. Trotzdem verloren auch wir schon einen Patienten nach einer transduodenalen extrahepatischen antegraden Dränage bei peritoneal metastasierendem Pankreaskarzinom mit Cholestase. Bei dem Patienten kam es am 4. postinterventionellen Tag zu einer kompletten Stentmigration in die Peritonealhöhle mit Entwicklung einer foudroyanten Peritonitis. Seit diesem Ereignis versuchen wir aufgrund der endoskopisch / röntgenologisch ersichtlichen Stentlage nach Freisetzung und in der US-Kontrolle die Stentdislokationsgefahr abzuschätzen. Bei Gefahr einer Stentdislokation (abgewinkelte Lage, kurzes endoluminals SEM-Segment im Intestinum, tangentialer Verlauf des SEM zum Intestinum ohne Adaptation von Intestinum und Leber resp. DHC) versuchen wir eine Dislokation durch Clipapplikation am luminalen Stentdurchtritt zu vermeiden, seither mit Erfolg. In der Industrie werden Anstrengungen in der Entwicklung moderner SEMs unternommen, um die Dislokationsgefahr zu vermindern. Erste Ansätze hierfür zeichnen sich bereits duch den Axios-Stent der Firma X-Luminal (Mountain View, CA, U.S.A.) ab.

\section{Technischer Erfolg und Langzeitverlauf}

Die in der Literatur bisher publizierten wenigen Langzeitdaten weisen klinische Erfolgsraten zwischen 66 und $90 \%$ auf $[3,5,6,45]$. Der technische Erfolg ist bereits während der Prozedur an der erreichten transluminalen Cholangiografie und der sicheren Stentplatzierung zu messen und mittels Fluoroskopie als auch endoskopisch-endoluminal mit Print oder Video zu dokumentieren. Daneben wird der (mittel- bis) langfristige Dränageerfolg durch
Besserung oder Verlust des Ikterus, verbunden mit einem gebesserten subjektiven Befinden und einer durch die Therapie der begleitenden Cholangitis verbesserten Lebensqualität, validiert.

Begleitende Bestimmungen der laborchemischen Cholestase(Bilirubin, alkalische Phosphatase) und Leberparameter (ALAT/ ASAT, $\gamma$-GT, GLDH) sowie der Entzündungsparameter helfen den klinischen EUCD-Erfolg zu objektivieren. Wurde unter palliativer Intention eine EUS-gestützte Gallenwegsdränage erreicht, sollten im weiteren Verlauf Reinterventionen nur bei klinischem oder laborchemischen Verdacht auf eine Reokklussion oder Progress der Grunderkrankung mit erneuter Cholestase durchgeführt werden. Die Frage, inwieweit eine erneute endoskopische oder -sonografische Intervention erforderlich ist und erfolgreich sein kann, ist idealerweise durch eine perkutane sonografische Untersuchung der Patienten zu klären, die großzügig, d.h. bei geringsten klinischen Verschlechterungen anberaumt werden sollte. Dies ist für die Patienten wenig aufwändig, nicht invasiv und hilft die Vertrauensbasis zwischen dem EUS-Therapeuten und dem Patienten zu festigen und zudem können rechtzeitig Komplikationen oder Dränageprobleme erkannt werden.

\section{Reintervention}

Einer der entscheidenden Vorteile der EUCD ist die prinzipielle Option einer endoskopischen Reintervention im Komplikationsbzw. Problemfall, die sich durch folgendes Profil in eigener klinischer Erfahrung auszeichnet:

- Replatzierung eines dislozierten Stents (Stentmigration),

- Stentwechsel (z. B. bei Stentokklusion),

- Stenttausch (Metall- gegen Plastikstent oder umgekehrt),

- Steinextraktion durch den Stent,

- Einlage weiterer Stents in den Gallengang durch den transhepatischen Zugang und

- endoskopische/-sonografische Interventionserweiterung je nach Grunderkrankung (z. B. Ileustherapie).

Im eigenen Patientenklientel von seinerzeit 52 systematisch interimsanalysierten Patienten wurden im weiteren „Follow-up“ nur in 4 Fällen Reinterventionen erforderlich, bei denen die Dränage erfolgreich korrigiert oder gewechselt wurde [45].

Dies spricht für die hohe Effizienz einer initial erfolgreichen EUCD und ihres Potenzials in der palliativen Behandlung des malignen Ikterus, denn eines der vordergründigsten Ziele in der Palliation ist es, nach Möglichkeit apparative interventionelle Reinterventionen zu vermeiden.

\section{Wissenschaftliche Bearbeitung} $\nabla$

Anhand jeweils zentrums- bzw. anwenderspezifischer klinischendoskopischer und -sonografischer Therapieerfahrungen sowie eines einschlägigen Literaturvergleiches wird in systematisch-wissenschaftlicher Weise im Rahmen von Einzelfallberichten, unizentrischen Fallserien oder multizentrisch zusammengestellten Behandlungskohorten die neuartige EUS-gestützte Cholangiodränage im therapeutischen Management konventionell-endoskopisch nicht therapierbarer Gallengangsstenosen beschrieben.

\section{Vorgehen}

Es werden alle konsekutiven symptomatischen Patienten mit Cholestase (definiert als Ikterus sowie 2-fach erhöhte Laborwerte Bilirubin und alkalische Phosphatase, ALT) in eine (anwendungs- 
begleitende) prospektive (möglichst multi-) unizentrische (ggf. sogar multinationale) systematische Beobachtungsstudie (Fallserie) über einen definierten Zeitraum einbezogen. Patienten-, befundund interventionsassoziierte Spezifika werden dann in computerbasierten Fallregistern dokumentiert. Die Machbarkeit wird mit dem technischen (erreichte Dränageplatzierung) und klinischen Erfolg (regressive Cholestase, angezeigt durch rückläufige Cholestase- und Entzündungsparameter/abnehmende Schmerzen, verbesserte Lebensqualität) charakterisiert. Das „Outcome“ wird bestimmt durch den klinischen Langzeiterfolg sowie Komplikationsspektrum und -raten (Häufigkeit der postinterventionellen Blutung, Perforation, Cholangitis, Schmerzen, Reintervention[srat] en, Notfalloperationen/-interventionen) und die Letalität inkl. der Analyse interventionsunabhängiger Todesfälle.

Zur Verifizierung des optimalen technischen Vorgehens wird indikations- und anatomieadaptiert ergebnisbeschreibend das Vorgehen und der Weg der Punktion bzw. der Dränageplatzierung:

- transesophageal / -gastral / -jejunal [46],

- Rendezvoustechnik,

transhepatisch intern antegrad,

- antegrad extrahepatisch (Choledocho-Enterostomie),

- retrograd intrahepatisch (Hepatiko-Enterostomie)

dargestellt, darunter v. a. jene in Rendezvoustechnik mit ERCP. Im Besonderen ist die Indikation zur EUCD deskriptiv zu differenzieren, meist Cholestase, diese jedoch durch

- Tumorrezidiv (z.B. Magenkarzinom nach vorheriger Gastrektomie; periampulläres Karzinom nach vorheriger pyloruserhaltender kephaler Pankreatoduodenektomie/OP nach KauschWhipple)

- Klatskin-Tumor und

- benigne Stenose oder Cholangiolithiasis bei Z.n. Hepaticojejunostomie oder B-II-Magenresektion oder Roux-en-Y-Anastomose mit endoskopisch nicht erreichbarer Anastomose oder Papille

bedingt.
Nicht zuletzt wird die relative Häufigkeit der Insertion der verschiedenen Stentdesigns, ob Metall- oder Plastikstent und ihre Größe (ca. 8,5-French, 10-French bzw. „double pigtail“) erfasst. Dies wird im Langzeitverlauf mit der Okklusions- und Stentmigrations- als auch Reinterventionsrate korreliert, um adaptiert für die jeweilige Indikation und spezielle Anatomie das optimale Stentdesign anzupassen.

Die Ergebnisse bisheriger, insgesamt sehr heterogener Studien sind in den Tab. 1, 2 wiedergegeben. Eine Vergleichbarkeit der Ergebnisse ist aufgrund erheblicher Unterschiede im Patientenkollektiv (Einschluss benigner und maligner Obstruktionen) und im technischen Vorgehen (Stentart, mit und ohne Bougierung) erheblich eingeschränkt.

Schlussfolgernd lässt sich aus der Analyse der Literaturdaten konstatieren, dass die transmurale EUS-gestützte Cholangiodränage (EUCD) bei entsprechender Expertise für ein selektioniertes Patientenklientel eine elegante und komplikationsarme Methode darstellt, die den therapeutischen Handlungsspielraum des interventionellen Endoskopikers suffizient erweitert. In klinischen Problemsituationen kann die EUCD eine alternative Ausweglösung darstellen und somit weit invasivere operative Interventionen vermeiden. Weiterführende systematische und prospektive Studien sind erforderlich, um generelle Therapieempfehlungen zu Indikation und periinterventionellem klinischen Management sowie zu einem differenzierten technischen Vorgehen ableiten zu können.

\section{Nachsorge}

Die postinterventionelle Betreuung wird entscheidend vom klinischen Zustand als auch der Lebenserwartung bei den meist palliativen Behandlungen aufgrund eines fortgeschrittenen oder metastasierten Tumorleidens bestimmt. Die transabdominelle Sonografiekontrolle am 1.postinterventionellen Tag erscheint inzwischen verbindlich. Hierbei gilt es bei entsprechender Klinik

Tab. 1 Publizierte Daten zur EUS-gestützten Cholangiodränage 2002 - 2008 (chronologische Reihenfolge).

\begin{tabular}{|c|c|c|c|c|c|}
\hline Autor & [n] & Stenttyp & techn. Erfolg & Komplikation & klin. Erfolg \\
\hline $\begin{array}{l}\text { Burmester et al. [7] } \\
\text { Gastrointest Endosc } \\
\text { 2003; 57: } 246-251\end{array}$ & 4 & Plaststent & $75 \%$ & 1 Peritonitis & k. A. \\
\hline $\begin{array}{l}\text { Püspök et al. [34] } \\
\text { Am J Gastroenterol } \\
\text { 2005; 100: } 1743-1747\end{array}$ & 6 & $\begin{array}{l}5 \text { Plaststent } \\
1 \text { SEMS }\end{array}$ & $100 \%$ & $\begin{array}{l}16 \% \\
\text { (1 Cholezystitis) }\end{array}$ & k. A. \\
\hline $\begin{array}{l}\text { Kahaleh et al. [35] } \\
\text { Gastrointest Endosc } \\
2006 ; 64: 52-59\end{array}$ & 23 & $\begin{array}{l}11 \text { Plaststent } \\
10 \text { SEMS }\end{array}$ & $91 \%$ & $\begin{array}{l}17 \% \\
\text { (1 Blutung, } 2 \text { Pneumo- } \\
\text { peritoneum, } 1 \text { Galleleck) }\end{array}$ & $78 \%$ \\
\hline $\begin{array}{l}\text { Bories et al. [4] } \\
\text { Endoscopy } \\
2007 ; 39: 287-291\end{array}$ & 11 & $\begin{array}{l}7 \text { Plaststent } \\
3 \text { SEMS }\end{array}$ & $90 \%$ & $\begin{array}{l}18 \% \\
\text { (1 Biliom, } 1 \text { Cholangitis) }\end{array}$ & $\begin{array}{l}90 \% \\
\text { (2 Reintervention) }\end{array}$ \\
\hline $\begin{array}{l}\text { Will et al. [33] } \\
\text { Endoscopy } \\
\text { 2007; 39: } 292-295\end{array}$ & 10 & $\begin{array}{l}4 \text { Plaststent } \\
5 \text { SEMS }\end{array}$ & $90 \%$ & $\begin{array}{l}12,5 \% \\
\text { (1 Blutung, } 1 \text { Cholangitis) }\end{array}$ & $80 \%$ \\
\hline $\begin{array}{l}\text { Tarantino et al. [36] } \\
\text { Endoscopy } \\
2008 ; 40: 336-339\end{array}$ & 8 & $\begin{array}{l}7 \text { Plaststent } \\
1 \text { Metall }\end{array}$ & $100 \%$ & $0 \%$ & $\begin{array}{l}100 \% \\
\quad(1 \text { Reintervention) }\end{array}$ \\
\hline $\begin{array}{l}\text { Yamao et al. [37] } \\
\text { Endoscopy } \\
2008 ; 40: 340-342\end{array}$ & 5 & Plaststent & $100 \%(5-C D)$ & 1 Pneumoperitoneum & $100 \%$ \\
\hline $\begin{array}{l}\text { Itoi et al. [38] } \\
\text { World J Gastroenterol } \\
\text { 2008; 14: } 6078-6082\end{array}$ & 4 & 4 Plaststent & $100 \%(C D)$ & $\begin{array}{l}25 \% \\
\text { (1 Blutung, Peritonitis) }\end{array}$ & $75 \%$ \\
\hline
\end{tabular}


Tab.2 Publizierte Daten zur EUS-gestützten Cholangiodränage 2009-2012 (chronologische Reihenfolge).

\begin{tabular}{|c|c|c|c|c|c|}
\hline Autor & [n] & Stenttyp & techn. Erfolg & Komplikationen & klin. Erfolg \\
\hline $\begin{array}{l}\text { Brauer et al. [3] } \\
\text { Gastrointest Endosc } \\
\text { 2009; 70: } 471-479\end{array}$ & 12 & Plaststent & $\begin{array}{l}92 \% \\
(7 \mathrm{R}, 4 \mathrm{CD})\end{array}$ & $\begin{array}{l}1 \text { Peritonitis } \rightarrow \text { OP } \\
1 \text { Pneumoperitoneum } \\
1 \text { pulmonale Insuffizienz }\end{array}$ & $75 \%(n=9 / 12)$ \\
\hline $\begin{array}{l}\text { Maranki et al. [6] } \\
\text { Endoscopy } \\
\text { 2009; 41: } 532-538\end{array}$ & 49 & $\begin{array}{l}\text { Metall } \\
\text { Plaststent }\end{array}$ & $84 \%$ & $16 \%$ & $\begin{array}{l}73 \% \text { intrahep. } \\
78 \% \text { extrahep. }\end{array}$ \\
\hline $\begin{array}{l}\text { Horaguchi et al. [39] } \\
\text { Dig Endosc } \\
\text { 2009; 21: 239-244 }\end{array}$ & 16 & Plaststent & $\begin{array}{l}94 \% \\
(8 \mathrm{CD}, 7 \mathrm{HD})\end{array}$ & $\begin{array}{l}12, \% \\
\text { (1 Peritonitis, } \\
16 \text { Stentmigration) }\end{array}$ & $\begin{array}{l}3 \text { Operation } \\
10 \text { Patienten mit } \\
\text { Stentwechsel }\end{array}$ \\
\hline $\begin{array}{l}\text { Park et al. [40] } \\
\text { Am J Gastrointerol } \\
\text { 2009; 104: } 2168-2174\end{array}$ & 14 & Metallstent & $100 \%$ & 2 Pneumoperitoneum & $93 \%$ \\
\hline $\begin{array}{l}\text { Hanada et al. [41] } \\
\text { Dig Endosc } \\
\text { 2009;21:S75-S78 }\end{array}$ & 4 & Plaststent & $100 \%$ & keine & $100 \%$ \\
\hline $\begin{array}{l}\text { Kim et al. [31] } \\
\text { Endoscopy } \\
\text { 2010; 42: } 496-502\end{array}$ & 15 & Plaststent & $\begin{array}{l}80 \% \\
\text { Rendezvous }\end{array}$ & keine & $80 \%$ \\
\hline $\begin{array}{l}\text { Ramirez-Luna et al. [5] } \\
\text { Endoscopy } \\
\text { 2011; 43(9): } 826-830\end{array}$ & 11 & Plaststent & $\begin{array}{l}91 \% \\
(8 \mathrm{CD}, 2 \mathrm{HD})\end{array}$ & $\begin{array}{l}18 \% \\
\text { (1 Biliom, } 1 \text { Stentmigration) }\end{array}$ & $\begin{array}{l}90 \% \\
\text { (1 Reintervent.) }\end{array}$ \\
\hline $\begin{array}{l}\text { Hara K et al. [43] } \\
\text { Am J Gastroent 2011; 106: } \\
\text { 1239-1245 }\end{array}$ & $18 \mathrm{pcs}$ & Plaststent & $\begin{array}{l}94 \% \\
(17 / 18 \mathrm{CD})\end{array}$ & $\begin{array}{l}2 \text { Peritonitis } \\
1 \text { Hämoblie }\end{array}$ & $\begin{array}{l}100 \% \\
\text { (12 Okklusionen; } \\
n=8,66 \% \text { Erfolg) }\end{array}$ \\
\hline $\begin{array}{l}\text { Siddiqui et al. [42] } \\
\text { Surg Endosc 2011; 25(2): } \\
549-555\end{array}$ & 8 & SEM & $\begin{array}{l}100 \% \\
(8 C D)\end{array}$ & $\begin{array}{l}1 \text { Perforation } \rightarrow \text { OP } \\
1 \text { Schmerzen }\end{array}$ & $100 \%$ \\
\hline $\begin{array}{l}\text { Komaki et al. [43] } \\
\text { Pancreatology } \\
\text { 2011; } 11: 47-51\end{array}$ & 15 & Plaststent & $\begin{array}{l}93 \% \\
(1 \mathrm{R}, 13 \mathrm{CD})\end{array}$ & $\begin{array}{l}2 \text { Peritonitis } \\
4 \text { Cholangitis } \\
1 \text { Stentmigration }\end{array}$ & $71 \%(n=10 / 14)$ \\
\hline $\begin{array}{l}\text { Will et al. [45] } \\
\text { Gastrointest Endosc } \\
\text { 2011; 73: AB } 257\end{array}$ & 52 & $\begin{array}{l}28 \text { SEMS } \\
11 \text { Plastst }\end{array}$ & $75 \%$ & $\begin{array}{l}3 \text { Cholangitis } \\
1 \text { Hämobilie } \\
1 \text { Peritonitis } \\
\text { 3 Stentdislokation(SEM) }\end{array}$ & $\begin{array}{l}90 \% \\
4 \text { Reintervent. }\end{array}$ \\
\hline $\begin{array}{l}\text { Eum et al. [44] } \\
\text { Gastrointest Endosc } \\
\text { 2010; 6: 1279-1284 }\end{array}$ & 4 & SEM & $\begin{array}{l}100 \% \\
\text { Pilotstudie } \\
\text { PDT, APC }\end{array}$ & keine & $100 \%$ \\
\hline $\begin{array}{l}\text { Park et al. [44] } \\
\text { Gastrointest Endosc } \\
\text { 2011; 74: 1267-1284 }\end{array}$ & 55 & $\begin{array}{l}\text { SEM } \\
\text { Plaststent }\end{array}$ & $96,5 \%$ & $\begin{array}{l}28 \% \\
\text { (2 Peritonitis, } 2 \text { Blutung, } \\
7 \text { Pneumoperit., } \\
7 \text { Stentmigration) }\end{array}$ & $89 \%$ \\
\hline $\begin{array}{l}\text { Shah et al. [30] } \\
\text { Gastrointest Endosc } \\
\text { 2012; 75: } 56-64\end{array}$ & 66 & $\begin{array}{l}\text { SEM } \\
\text { Plaststent }\end{array}$ & $\begin{array}{l}50 \text { Rendezv. } \\
\text { Erfolg: } 74 \% \\
16 \text { HD: } 81 \%\end{array}$ & $\begin{array}{l}10 \% \\
\text { (Leckage, Bakteriämie, } \\
\text { Pankreatitis) }\end{array}$ & k. A. \\
\hline
\end{tabular}

nach interventionsassoziierten intraabdominellen Flüssigkeitsansammlungen (ursächlich Blutung, Galle, Inflammation) zu fahnden. Eine persistente Cholestase, eine fehlende Aerobilie, ein nicht erkennbarer Stent als auch die Zunahme freier Flüssigkeit im Vergleich zur Sonografie vor EUCD sind im Zusammenhang mit Schmerzen ein Warnsignal und sollten umgehend weitere diagnostische und therapeutische Maßnahmen veranlassen (diagnostische Punktion des Aszites [Galleleckage], CT, PTCD). Periodische Untersuchungsintervalle und das dann zu empfehlende Spektrum (klinische Untersuchung, Laborprofil, Bildgebung [transabdominale/endoskopische Sonografie, Endoskopie]) sind Gegenstand des derzeitigen klinischen Erfahrungserwerbs (s. u. Kapitel „Ausblick“). Eine umgehende klinische, laborchemische und bildgebende Kontrolle des EUCD-ausführenden Zentrums im Falle von Problemen (AZ-Verschlechterung, Fieber, Schmerzen, Zunahme des Ikterus etc.) ist dringend anzuraten.

\section{Diskussion}

Die erste erfolgreiche EUS-gestützte Cholangiodränage wurde von Giovannini im Jahr 2001 ausgeführt [47].

Die EUS-gestützte Intervention am Gallenwegsystem (EUCD) als auch am Pankreasgang (EUPD) sind erstmals als Fallberichte 2001 und 2003 sowie nachfolgend in kleinen Fallserien publiziert worden $[1,2,10,11,47-49]$.

Damit reiht sich die EUCD in das neue Profil interventionell-endosonografischer Prozeduren ein, die sich mittlerweile neben der transluminalen Angehbarkeit des Pankreasgangs [2, 11, 30, 48 ] z.B. auch auf (post-)inflammatorische Komplikationen des Pankreas wie Pseudozysten, Abszesse und infizierte Nekrosen im Rahmen einer nekrotisierenden Pankreatitis (EUS-gestützte Dränage, endoskopisches Debridement) erstrecken [14]. 
Im Gegensatz zur PTCD, die eine Morbidität von 5-20\% aufweist und die in $5-10 \%$ anstatt einer extern-internen lediglich nur eine externe Dränage erreicht, erlaubt die EUS ein minimalinvasives Vorgehen mit einer primären internen biliären Dränage, eine niedrige bis wägbare Komplikationsrate und die Möglichkeit einer endoskopischen /-sonografischen Reintervention bei Dränageproblemen $[5,19,20]$.

Als eine Voraussetzung für EUS-gestützte transluminale Interventionen gilt die etablierte und weithin genutzte, EUS-gestützte Feinnadelpunktion [29, 50]. Mit der ersten EUS-gestützten Cholangiografie wies Wiersema im Jahr 1996 auf die Möglichkeit hin, dass auch das Gallenwegsystem für EUS-Interventionen angehbar ist [51].

In einer ersten kleineren Serie an 4 Patienten konnte Burmester 2003 zeigen, dass auch im Langzeitverlauf beeindruckende Ergebnisse zu erzielen sind [7].

Die Indikationen zur EUCD halten sich auch in Zentren der spezialisierten endoskopischen Intervention mit hoher ERC-Expertise in Grenzen. Um die Häufigkeit zu charakterisieren, sind die endoskopischen Zahlen beispielhaft im Verhältnis der verschiedenen Methoden über einen 3-Jahres-Zeitraum in der anfänglichen klinischen Etablierungsphase (2002 - 2005) der EUCD aufgeführt [33].

\begin{tabular}{|ll|}
\hline Ösophagogastroskopie & $\mathrm{n}=17857$ \\
\hline EUS & $\mathrm{n}=2371$ \\
\hline interventionelle EUS & $\mathrm{n}=457$ \\
\hline ERCP & $\mathrm{n}=2287$ \\
\hline PTCD & $\mathrm{n}=36$ \\
\hline EUCD & $\mathrm{n}=8$ \\
\hline
\end{tabular}

In den folgenden Jahren 2005-2010 wurden in unserer Abteilung weitere 44 Patienten mit der EUS-gestützten Dränage behandelt [45]. Die Zunahme der EUCD ist in erster Linie in der Indikationsausweitung und unserer Präferenz der EUCD vor PTCD als auch in der direkten Zuweisung aus anderen Kliniken speziell zur EUCD bei Problemen mit der PTCD oder frustraner ERC zu sehen. Dennoch nimmt sich eine derzeitige jährliche Untersuchungsfrequenz an unserem Zentrum von 20 Patienten, bei denen eine EUCD ausgeführt wird, sehr bescheiden aus und soll Hinweis sein, dass selbst in einem Zentrum die Expertise nicht auf breiter Front aufzubauen ist. Aufgrund der begrenzten Indikation, der erforderlichen hohen interventionellen Expertise und der langen Lernkurve sollte die EUCD ausschließlich in hochspezialisierten endoskopisch-endosonografischen Zentren vorgehalten werden.

Die Vorgehensweisen in den bisher beschriebenen Fallserien sind daneben nur partiell vergleichbar. Das Methodenspektrum und die Techniken haben sich in den letzten 5 Jahren außerdem erheblich erweitert. Neben einer Rendezvoustechnik werden die antegrade interne Dränage und die antegrade Choledocho-Enterostomie sowie die retrograde Hepatico-Enterostomie beschrieben. Die Techniken wurden an die anatomischen Variationen (Papille erreichbar, Z.n. Operation) adaptiert [1 - 7, 30, 44].

Sollte man eine Wichtung bezüglich des empfohlenen Vorgehens durchführen, würde, diese wie folgt, zu beschreiben sein:

Ist eine ERC indiziert, die aber aufgrund einer destruierten Papille oder eines nicht zu sondierenden DHC frustran bleibt, sollte in gleicher Sitzung ein EUS-geführtes Rendezvous versucht werden. Gelingt nach Punktion die Ausleitung des Drahtes, kann nach Gerätewechsel eine konventionelle ERC durchgeführt werden. Handelt es sich um einen Patienten mit nicht erreichbarer Papille, kann nach Punktion und Cholangiografie eine antegrade interne
Dränage versucht werden. Voraussetzung hierfür sind eine Drahtpassage der Stenose und ein offenes intestinales Lumen „hinter“ dem Tumor. Gelingt die Passage des Tumors nicht, kann je nach initialer Punktion (D.hepaticus, D.choledochus) eine retrograde transhepatische Hepatico-Intestinostomie oder eine antegrade extrahepatische Choledocho-Intestinostomie versucht werden.

Die Untersuchung beginnt in den meisten Fallserien mit einer Punktion der gestauten intra- oder extrahepatischen Gallenwege mit einer 19-G-Nadel. Nach Drahtvorschub werden nunmehr alternativ Bougies von $5-7$ French und nachfolgend 4-mm- oder 6-mm-Dilatationsballons eingesetzt oder über den Draht ein HFRingmesser vorgeführt. Letztere Methode wurde von uns inauguriert und wird im klinischen Alltag bevorzugt. Diese hat den Vorteil, dass in den meisten Fällen auf lumenerweiternde Dilatationen verzichtet werden kann, da 8,5-French-Pigtailprothesen oder ein gecoverter Metallstent den präformierten Entero-Biliostomie-Kanal gut passieren. Durch die Applikation von HF-Koagulationsstrom wird zudem das Blutungsrisiko minimiert.

In den fast 10 Jahren eigener Erfahrungen mit der EUCD stellt sich als eine deletäre Komplikation der EUCD die peritoneale Galleleckage heraus. Da diese bei häufig infizierter Galle als Hauptursache für eine Peritonitis und damit für die Morbidität und Letalität der Methode verantwortlich ist, gilt es, die Leckage zu vermeiden, indem:

- die Prozedur patientenadaptiert nach technischen Algorithmen erfolgt,

- der transintestinale Wandverschluss nach EUS-Intervention garantiert wird und

- durch Einsatz spezieller Stents und deren Sicherung dessen Dislokation oder Migration verhindert wird.

In den bisher mehr als 200 publizierten Fällen wurde über Komplikationsraten von im Mittel 15 (Streubreite: 0-25)\% berichtet. Somit kann die Methode im Vergleich zur PTCD als komplikationsärmer und relativ sicher eingeschätzt werden. Bisher existiert nur eine prospektive Studie [38], die einen technischen Erfolg von $94 \%$ bei Anlage einer Choledocho-Duodenostomie erreichen konnte. Neben einer Hämobilie wird über 2 Patienten berichtet, die eine Peritonitis entwickelten (Komplikation: 17\%). Im Langzeitverlauf traten bei 12/17 Patienten Stentokklussionen der Plastikstents auf, die in 66\% erfolgreich behandelt wurden. Die Studie zeigt eindrucksvoll, dass die EUCD zwar eine elegante Methode der alternativen Cholangiodränage darstellt, deren technische Durchführung jedoch in weiteren Studien evaluiert und verbessert werden muss, um die Komplikationsraten und Reinterventionsraten zu minimieren.

\section{Ausblick}

$\nabla$

- Der gegenwärtig erreichte weltweite Stand von ca. 200 Fällen mit EUCD legt die Idee nahe, mittels (inter-)nationalem Fallregister eine systematische Erfassung der versorgten Fälle durch die Nestoren, Inauguratoren und Schrittmacher der Methode zu initiieren, um durch Bündelung der erzielten Erfahrungen aus der klinischen Praxis eine kompetente Indikationsstellung zu vermitteln und in der klinischen Praxis durchsetzen zu helfen. Die multizentrische (/-nationale) Falldokumentation im Rahmen einer prospektiven klinischen systematischen Beobachtungsstudie (s. o. Kapitel „Wissenschaftliche Be- / Aufarbeitung") ist dazu ein suffizienter und kompetenter erster Schritt einer wissenschaftlichen Aufarbeitung und kann durch ihre 
systematische Weiterverfolgung zu einer neuen Aussagequalität, insbesondere mit größeren Fallzahlen gelangen.

- Ob „Interventionelle EUS-(EUCD-)Kurse für Fortgeschrittene“ der Methode zu einer umsichtigen Verbreitung verhelfen, muss abgewartet werden. Von großem Interesse werden in diesem Zusammenhang die sich ergebende Lernkurve und ihre Konsequenzen sein. Derzeit kann eine EUS-gestützte Dränage nur für Zentren mit hoher Expertise in der interventionellen EUS unter Vorhaltung komplementärer Methoden zur Cholangiodränage (PTCD, viszerale Chirurgie, interventionelle Radiologie) empfohlen werden.

- Eine interessante Problemstellung betrifft den Umstand der Indikationsausweitung auf selektive benigne Fallkonstellationen (Anastomosenstenose nach Hepaticojejunostomie, Cholangiolithiasis nach BII-Magenresektion, Roux-en-Y-Anastomose), die bei verantwortungsvoller Indikationsstellung in gemeinsamer Entscheidungsfindung mit dem Patienten bereits heute Anwendung finden.

Da es bisher keine vergleichende Studie mit der PTCD als derzeitigen Goldstandard nach erfolgloser ERC bei Cholestase gibt, bleibt abzuwarten, ob die EUS-gestützte Cholangiodränage ihre derzeitige Präferenz als experimentielle Therapie in der Hand des Spezialisten verlassen und in breiter Front als Therapie der ersten Wahl nach erfolgloser ERC Anwendung finden kann. Hierzu bedarf es in den Curricula der Ausbildung zum interventionellen Endoskopiker resp. Endosonografiker aus unserer Sicht erheblicher Anstrengungen, um die komplikationsarme EUCD flächendeckend anbieten zu können. Ein unkritischer Einsatz der Methode von in der EUS-gestützten Intervention wenig erfahrenen Kollegen in Zentren mit eingeschränkter viszeralchirurgischer und interventionell-radiologischer Kompetenz birgt die Gefahr in sich, den oft schwerkranken Patienten durch die EUSbasierte Intervention weiteren Schaden zuzufügen. Dies muss im Interesse der uns anvertrauten Patienten und der Methode dringend vermieden werden.

\section{Fazit}

$\nabla$

Die EUS-gestützte transluminale (-gastrale, -jejunale, -ösophageale [52]) Cholangiodränage mit antegrader oder retrograder Galleableitung oder in Rendezvoustechnik ist im ausgewählten Fall eine

- dem Anspruch von NOTES durchaus genügende,

$\checkmark$ elegante,

sinnvolle,

- in Bezug auf die erforderliche Expertise technisch machbare,

- hinsichtlich der Komplikationsrate akzeptabel sichere,

- klinisch noch experimentelle aber

- ermutigende und

- vielversprechende,

- hinsichtlich des periinterventionellen Risikos kalkulierbare bzw. tolerable

neue Methode der interventionellen Endoskopie. Die EUCD sollte präferenziell bei maligner Cholestase und frustraner ERC aufgrund von anatomischen Besonderheiten (postoperativ veränderte Anatomie des oberen GI-Trakts), tumorbedingten Stenosierungen (nicht erreichbarer/sondierbarer Papille) und Kontraindikationen oder Problemen der PTCD in Erwägung gezogen werden. In erfahrenen Zentren der EUS-Intervention kann die Methode der EUCD eine primäre PTCD nach erfolgloser ERC ersetzen.
Die Intervention sollte durch einen sehr erfahrenen interventionellen Endoskopiker /-sonografiker eines Zentrums durchgeführt werden. Unabdingbar für die Durchführung der EUCD ist die Kompetenz eines im chirurgisch-operativen Management endoskopischer Komplikationen erfahrenen Chirurgen bzw. eines erfahrenen interventionellen Radiologen, um im Falle von Komplikationen unmittelbar und adäquat intervenieren zu können.

Dies ist eine conditio sine qua non, um die Eingriffsmorbidität zu minimieren und mögliche Komplikationen zielgerichtet und effektiv zu behandeln.

Als Vorteile der EUCD sind

- die limitierte Invasivität (Vermeidung des erheblichen OPTraumas),

- die permanente interne Dränage,

- die Erreichung eines zügigen klinischen Effekts der Gallenwegsentlastung, als auch

- die weiterbestehende Option einer aussichtsreichen, meist recht unproblematischen, d.h. nochmaligen endoskopisch/ -sonografischen Reintervention und

- die Erweiterung des therapeutischen Vorgehensspektrums

zu nennen.

Weiterführende systematische Untersuchungen, insbesondere hinsichtlich der klinischen Alltagsanwendbarkeit nach Überwindung der singulären, fallspezifischen klinischen Einführung im Sinne der klinisch-systematischen prospektiven Observationsstudie, ggf. in simultaner fallunabhängiger methodischer Gegenüberstellung zu ERC und PTCD oder historischem Vergleich zu diesen Methoden, insbesondere ausgerichtet auf ein engmaschiges mittel- bis langfristiges Follow-up, erscheinen empfehlenswert.

Dies hilft den allgemeinen Erfahrungswert zu erhöhen, insbesondere im Hinblick auf Komplikationen oder fatale Verläufe.

Eine entsprechende Analyse erlaubt es, Präventionsstrategien zu entwickeln, um Komplikationen zu vermeiden. Eine akribische Aufarbeitung der Techniken und der Ergebnisse der EUCD ist erforderlich, um die anspruchsvolle endoskopische Intervention perspektivisch in Richt- oder Leitlinienrelevanz zu überführen.

\section{Interessenkonflikt: Nein}

\section{Literatur}

1 Kahaleh M, Wang P, Shami VM et al. EUS-guided transhepatic cholangiography: report of 6 cases. Gastrointest Endosc 2005; 61: 307-313

2 Will U, Fueldner F, Goldmann B et al. Successful transgastric pancreaticography and endoscopic ultrasound-guided drainage of a disconnected pancreatic tail syndrome. Ther Adv Gastroenterol 2010; 4: 213-218 DOI: 10.1177/1756283X10394232

3 Brauer BC, Chen YK, Fukami N et al. Single-operator EUS-guided cholangiopancreatography for difficult pancreaticobiliary access (with video). Gastrointest Endosc 2009; 70: 471-479

4 Bories E, Pesenti C, Caillol F et al. Transgastric endoscopic ultrasonography-guided biliary drainage: results of a pilot study. Endoscopy 2007; 39: $287-291$

5 Ramirez-Luna MA, Tellez-Avila FL, Giovannini M et al. Encoscopic ultrasound-guided biliodigestive drainage is a good alternative in patients with unresectable cancer. Endoscopy 2011; 43: 826-830

6 Maranki J, Hernandez AJ, Arslan B et al. Interventional endoscopic ultrasound-guided cholangiography: long-term experience of an emerging alternative to percutaneous transhepatic cholangiography. Endoscopy 2009; 41: 532-538

7 Burmester E, Niehaus J, Leineweber T et al. EUS-cholangio-drainage of bile duct: report of 4 cases. Gastrointest Endosc 2003; 57: 246-251

8 Will $U$, Wanzar C, Gerlach $R$ et al. Interventional ultrasound-guided procedures in pancreatic pseudocysts, abscesses and infected necroses - treatment algorithm in a large single-center study. Eur J Ultrasound (Ultraschall Med) 2011; 32: 176-183 
9 Seewald S, Ana TL, Teng KC et al. EUS-guided drainage of pancreatic pseudocysts, abscesses and infected necrosis. Dig Endosc 2009; 21 (Suppl. 01): 61-S65

10 Kahaleh M, Yoshida C, Yeaton P. EUS antegrade pancreatography with gastropancreatic duct stent placement. Review of two cases. Gastrointest Endosc 2003; 6: 919-923

11 Will U, Fueldner F, Thieme A et al. Transgastric pancreaticography and EUS-guided drainage of the pancreatic duct. J Hepatobiliary Pancreat Surg 2007; 14: 377 - 382 DOI: 10.1007/s00534-006-1139-8

12 Tessier G, Bories E, Arvanitakis $M$ et al. EUS-guided pancreatogastrostomy and pancreatobulbostomy for the treatment of pain in patients with pancreatic ductal dilatation inaccessible for transpapillary endoscopic therapy. Gastrointest Endosc 2007; 65: 233 - 241

13 Ergun M, Aouattah T, Gillain C et al. Endoscopic ultrasound-guided transluminal drainage of pancreatic duct obstruction: long-term outcome. Endoscopy 2011; 43: 518-525

14 Will U, Müller A, Wanzar I et al. Therapeutische endosonographische Drainagen - Indikationen und Technik. Endo heute 2011; 24: 33-50 DOI: http://dx.doi.org/10.1055/s-0031-1271357

15 Fogel EL, Shermann S, Devereaux BM et al. Therapeutic biliary endoscopy. Endoscopy 2001; 33: $31-38$

16 Schofl R. Diagnostic endoscopic retrograde cholangiopancreatography. Endoscopy 2001; 33: 147-157

17 Carr-Locke DL. Overview of the role of ERC in the management of diseases of the biliary tract and the pancreas. Gastrointest Endosc 2002; 56: $157-160$

18 Ferrucci JT Jr, Mueller PR, Harbin WP. Percutaneous transhepatic biliary drainage: technique, results and applications. Radiology 1980; 135: $1-13$

19 Harbin WP, Mueller PR, Ferrucci JT Jr et al. Transhepatic cholangiography: complications and use patterns of the fine-needle technique: a multiinstitutional survey. Radiology 1980; 135: 15-22

20 Calvo MM, Bujanda L, Heras I et al. The rendezvous technique for the treatment of choledocholithiasis. Gastrointest Endosc 2001; 54: 511 513

21 Keck T. [Carcinoma of the pancreas: current status of multimodal therapy]. Zentralbl Chir 2011; 136: 352-358

22 Hüser N, Assfalg V, Michalski CWet al. [Unresectable pancreatic cancerpalliative interventional and surgical treatment]. Zentralbl Chir 2010 135: $502-507$

23 Schütte K, Weigt J, Meyer F et al. Palliativtherapie im Grenzgebiet zwischen Gastroenterologie und (Viszeral-) Chirurgie. Zentralbl Chir 2010; 135: 528-534

24 Warnick P, Bahra M, Andreou A et al. Pankreasmalignom: Second-LookOperation trotz auswärtiger Inoperabilität? Zentralbl Chir 2010; 135: $0170-74$

25 Calasan I, Junger M, Schwendtner $P$ et al. Pankreaschirurgie an einem Krankenhaus der Grund- und Regelversorgung. Zentralbl Chir 2009; 134: $160-165$

26 Seifert $H$, Wehrmann $T$, Hilgers $R$ et al. Catheter probe extraductal EUS reliably detects distal common bile duct abnormalities. Gastrointest Endosc 2004; 60: 61 - 67

27 Varadarajulu S, Phadnis MA, Christein JD et al. Multiple transluminal gateway technique for EUS-guided drainage of symptomatic walledoff pancreas necrosis. Gastrointest Endosc 2011; 74: 74-80

28 Varadarajulu S, Bang JY, Phadnis MA et al. Endoscopic transmural drainage of peripancreatic fluid collections: outcomes and predictors of treatment success in 211 consecutive patients. J Gastrointest Surg 2011; $15: 2080-2088$

29 Will U, Mueller A, Topalidis T et al. Value of endoscopic ultrasonography (EUS)-guided fine needle aspiration (FNA) in the diagnosis of neoplastic pancreatic tumor(-like) pancreatic lesions in daily clinical practice. Ultraschall Med (Eur J Ultrasound) 2010; 31: 169-174 DOI: 10.1055/ s-0028-1109491

30 Shah JN, Marson F, Weilert F et al. Single-operator, single-session EUSguided anterograde cholangiopancreatography in failed ERCP or inaccessible papilla. Gastrointest Endosc 2012; 75: 56-64

$31 \mathrm{Kim}$ S, Gupta K, Mallery S et al. Endoscopic ultrasound rendezvous for bile dict access a transduodenal approach: cumulative experience at a single center: A cases series. Endoscopy 2010; 42: 496-502

32 Nguyen-Tang T, Binmoeller KF, Sanchez-Yague A et al. Endoscopic ultrasound (EUS)-guided transhepatic anterograde self-expandable metal stent (SEMS) placement across malignant biliary obstruction. Endoscopy 2010; 42: 232-236

33 Will $U$, Thieme Annakatrin, Gerlach $R$ et al. Treatment of biliary obstruction in selected patients by endoscopic ultrasonography (EUS)guided transluminal biliary drainage. Endoscopy 2007; 39: 292-295 PMID: 17357950

34 Püspök A, Lomoschütz F, Dejaco C et al. Endoscopic ultrasound guided therapy of benign and malignant biliary obstruction: a case series. Am J Gastroenterol 2005; 100: 1743 - 1747

35 Kahaleh M, Hernandez AJ, Tokar J et al. Interventional EUS-guided cholangiography: evaluation of a technique in evolution. Gastrointest Endosc 2006; 64: $52-59$

36 Tarantino I, Barresi L, Repici A et al. EUS-guided biliary drainage: a case series. Endoscopy 2008; 40: 336-339

37 Yamao K, Bhatia V, Mizuno $N$ et al. EUS-guided choledochoduodenostomy for palliative biliary drainage in patients with malignant biliary obstruction: results of long-term follow-up. Endoscopy 2008; 40: $340-342$

38 Itoi T, Itokawa F, Sofuni A et al. Endoscopic ultrasound-guided choledochoduodenostomy in patients with failed endoscopic retrograde cholangiopancreatography. World J Gastroenterol 2008; 14: 6078 6082

39 Horaguchi J, Fujita N, Noda Y et al. Endosonography-guided biliary drainage in cases with difficult transpapillary endoscopic biliary drainage. Dig Endosc 2009; 21: 239-244

40 Park do H, Koo JE, Oh J et al. EUS-guided biliary drainage with one-step placement of a fully covered metal stent for malignant biliary obstruction: a prospective feasibility study. Am J Gastroenterol 2009; 104: $2168-2174$

41 Hanada K, Iiboshi T, Ishii Y. Endoscopic ultrasound-guided choledochoduodenostomy for palliative biliary drainage in cases with inoperable pancreas head carcinoma. Dig Endosc 2009; 21 (Suppl. 01): S75-S78

42 Siddiqui AA, Sreenarasimhaiah J, Lara LF et al. Endoscopic ultrasoundguided transduodenal placement of a fully covered metal stent for palliative biliary drainage in patients with malignant biliary obstruction. Surg Endosc 2011; 25: 549-555

43 Komaki T, Kitano M, Sakamoto $\mathrm{H}$ et al. Endoscopic ultrasonographyguided biliary drainage: evaluation of a choledochoduodenostomy technique. Pancreatology 2011; 11 (Suppl. 02): 47-51

44 Park DH, Jang JW, Lee SS et al. EUS-guided biliary drainage with transluminal stenting after failed ERCP: predictors of adverse events and long-term results. Gastrointest Endosc 2011; 74: 1276-1284

45 Will $U$, Mueller AK, Kern $C$ et al. Differential Treatment of Biliary Obstructions With the Alternative EUS-Guided Translumenal Cholangiodrainage (EUCD) in Case of Impossible ERC - Representative Case Series ( $=52$ ). Gastrointest Endosc 2011; 73 Suppl: AB 257

46 Will U, Meyer F, Schmitt W et al. Endoscopic ultrasound-guided transesophageal cholangiodrainage and consecutive transhepatic Wallstent insertion into a jejunal stenosis. Scand J Gastroenterol 2007; 42: 412 415 DOI: $10.1080 / 003655206008811360036-5521$ print 1502-7708 online

47 Giovannini M, Moutardier V, Pesenti $C$ et al. Endoscopic ultrasoundguided bilioduodenal anastomosis: a new technique for biliary drainage. Endoscopy 2001; 33: 898-900

48 Giovannini M, Dotti M, Bories $E$ et al. Hepaticogastrostomy by echoendoscopy as a palliative treatment in a patient with metastatic biliary obstruction Endoscopy 2003; 35: 1076-1078

49 Mallery S, Matlock J, Freeman ML. EUS-guided rendezvous drainage of obstructed biliary and pancreatic ducts: report of 6 cases. Gastrointest Endosc 2004; 59: 100-107

50 Byrne MF, Gerke H, Mitchell RM et al. Yield of endoscopic ultrasoundguided fine-needle aspiration of bile duct lesions. Endoscopy 2004; 36: $715-719$

51 Wiersema MJ, Sandusky D, Carr R et al. Endosonography-guided cholangiopancreatography. Gastrointest Endosc 1996; 43: 102-106

52 Eum J, Park do H, Ryu CH et al. EUS-guided biliary drainage with a fully covered metal stent as a novel route for natural orifice transluminal endoscopic biliary interventions: a pilot study (with videos). Gastrointest Endosc 2010; 72: 1279-1284

53 Hara K, Yamao K, Niwa Y et al. Prospective clinical study of EUS-guided choledochoduodenostomy for malignant lower biliary tract obstruction. Am J Gastroenterol 2011; 106: 1239-1245 